\title{
The relevance of rapid assessment to health research and interventions
}

\author{
CAROL VLASSOFF' ${ }^{1}$ AND MARCEL TANNER ${ }^{2}$ \\ 'Special Programme for Research and Training in Tropical Diseases, World Health Organization, \\ Geneva, Switzerland and 2Department of Public Health and Epidemiology, Swiss Tropical Institute, \\ Basel, Switzerland
}

\begin{abstract}
This paper introduces the special issue on rapid assessment methods for tropical disease research. It highlights disease control problems that the combined skills of social and biomedical scientists must address, as well as problems hampering multidisciplinary research. Traditional social science and epidemiological methods are of ten too time-consuming to address the immediate and urgent needs of disease control programmes. Rapid assessment methods to provide information on health status, health impact, health services and health behaviour are therefore essential. A distinction is made between the efficacy of disease control tools and community effectiveness (the efficacy of the tools as applied at the community level), and the potential for rapid assessment to improve community effectiveness is emphasized. The development and validation of rapid assessment methods is discussed, and the techniques described more fully in subsequent papers are introduced.
\end{abstract}

\section{Introduction}

The medical doctors were ecstatic! Finally a drug had been developed that could cure hiccupalitis, a parasitic disease that produced an unusual complex of symptoms including continual hiccuping and memory loss. The disease afflicted mainly rural populations because it was transmitted by Sibophelus lamiensis - a fly which was sylvatic in habitat. However, trucks and rural migrants were apparently transporting the vectors to the city, and urban dwellers were increasingly contracting the disease. As the vector resembled the common house fly, people found it difficult to believe that this apparently familiar pest was a serious threat to their health. Prevention through vector control was therefore a losing battle, but researchers had finally come up with a drug that promised to solve the problem.

The new drug was provided free of charge and distributed to hospitals and medical services throughout endemic countries. Within a few weeks hiccupalitis was under control in the urban areas. Unfortunately, it continued to menace rural districts. Although clinical records indicated that the drug had been prescribed for all known patients, new cases of hiccupalitis con- tinued to appear. Apparently, a sizeable population was failing to report the disease and therefore not receiving treatment.

Clearly, the only alternative was to find out more about the people, their behaviour, and that of the health care providers. A sociologist and an anthropologist were called in to resolve the problem. Answers were needed quickly, because reinfection was being reported in treated areas where the disease was believed to be under control. The social scientists knew little about the disease - its causes, modes of transmission and treatment and they had never worked with a medical team. But, being well trained in survey methods, they designed a questionnaire on knowledge, attitudes, practices, and a range of sociocultural factors. Interaction with disease control personnel was minimal - the social scientists, busy with the survey, had little time for consultation. Moreover, they were loath to reveal their ignorance about the disease to their medical colleagues. The health personnel likewise ignored the social scientists. They failed to see the relevance of the survey to disease control and were worried because it was taking so long but, not understanding social science methods, they hesitated to interfere. 
Carol Vlassoff and Marcel Tanner

Two years later the social scientists completed a report containing an elaborate theoretical framework, an exhaustive literature review, and complex statistical results. The final section provided findings and recommendations concerning noncompliance with the new drug. It showed, for example, that in some communities the health workers had instructed patients to take a pill every four hours for three days. But patients suffering from acute memory loss as a result of the disease had failed to remember to take it. The health workers had not been forewarned about this eventuality, or about the importance of soliciting the help of family members or friends. In another area, holy powers were thought to be manifested by strange vocal utterances. Hiccupping was therefore considered a spiritual visitation and efforts to interfere with these voices were adamantly rejected by the community.

In spite of the significance of these insights for hiccupalitis control, little use was made of the report. Health personnel failed to read beyond the first few chapters, none of which contained information they considered relevant to their needs. The social scientists published their findings in international journals, but did not discuss them with the control programmes. Nor were they invited to do so. Unfortunately hiccupolitis continues to plague the populations of many developing countries. Scientists are busy searching for a better drug since resistance is now a serious problem in most endemic areas.

The above narrative is purely fictional, but it illustrates real problems and challenges for community-based research on health and development issues. In particular, it illustrates the tasks for the social and economic research component (SER) of the Special Programme for Research and Training in Tropical Diseases (TDR) of the World Health Organization (WHO). Recognizing the importance of human behaviour in the control of the diseases covered by TDR's programme - malaria, schistosomiasis, the filariases (including onchocerciasis), the trypanosomiases (including both African sleeping sickness and Chagas' disease), the leishmaniases and leprosy - SER was established in 1979. The incorporation of social and economic research in the early days of TDR's evolution was a milestone in communicable diseases research, an area in which human factors had, until then, generally been regarded as secondary to the understanding of vector and parasite behaviour.

SER has encountered many obstacles in implementing its mandate. This being a new field of study, social and economic research has sometimes suffered from poorly developed methodologies. It has also been criticized for being too theoretical and of little practical use to disease control programmes. Social scientists reply that they are typically consulted as a 'last resort' to offer 'quick' but often impossible solutions when other alternatives have failed. Moreover, today's developing country contexts in which field research is conducted often lack adequate facilities and trained personnel. Sometimes too, poor cooperation from disease control personnel prevents social scientists from understanding the disease and the concerns of control programmes.

Scientific rigour also requires that often, studies take too long to meet the immediate needs of disease control programmes. Thus, there is a need for more rapid methods of assessing high risk situations or diagnosing urgent problems. While rapid assessment methods are becoming increasingly popular, they tend to be diverse and often poorly articulated. For this reason, we present, in this issue, an outline of a number of procedures currently being explored by TDR. In some cases, the methods are being implemented on a pilot basis to investigate their applicability and utility in tropical disease research. In other cases, we take a critical look at the potential strengths and weaknesses of rapid assessment methods used elsewhere in the health field, but not yet applied to the target diseases of TDR.

\section{The status of rapid assessment}

Rapid assessment methods are an essential basis for the translation of research results into disease control activities. They can provide information for all categories of data required in health care and service management, notably:

- Health status information: frequency, distribution, causes/determinants of morbidity and mortality

- Health impact information: efficacy and effectiveness of policies, strategies and programmes

- Health service information: availability, supply, utilization and cost of services 
- Health behaviour information: health-seeking and risk-taking behaviour of individuals and/ or communities, determinants of behaviour, need and demand patterns.

A short review of the developments over the past years in the field of communicable disease control and in primary health care (PHC) implementation will place rapid assessment methods within the context of health and disease, and particularly disease control.

Extremely important advances have taken place in the diagnosis, prophylaxis and therapy of communicable disease agents. Novel serological tests, the development of simple field tests in parasitology and serology and the application of gene probes, have substantially improved the efficacy of clinical and community diagnosis. In addition, new, efficacious and safe drugs such as praziquantel, ivermectin and the benzimidazole compounds have offered new methods of drug therapy at the individual and community level. In addition, great efforts have been made, and considerable success achieved, in the improvement of existing vaccines and the development of new ones. Finally, advances in vector control tools and strategies, such as impregnated bednets for malaria control and the work of the Onchocerciasis Control Project (OCP) have provided efficacious tools for national or regional control programmes.

On the other hand, the PHC concept was adopted by numerous countries which designed national strategies around it. After an initial focus on selective strategies, for example, mother and child health programmes, immunization, essential drugs and nutritional supplementation approaches (where the outcome was mainly assessed by cost-effectiveness criteria), more attention was paid to integrated communitybased approaches in order to reach and ensure sustainability of the activities. Therefore, there is a move towards decentralization of health care management, based on existing services (governmental, non-governmental and private). Health services management at the district level based on the concept of the health district (WHO 1988) appears as an efficient strategy.

This brief summary of the evolution and the state of disease control within PHC shows that there is now a greater concern to improve community effectiveness rather than to focus exclusively on increasing the efficacy of disease control tools. This is illustrated by two hypothetical examples in Figures $1 \mathrm{a}$ and $1 \mathrm{~b}$. In Figure $1 \mathrm{a}$ we follow the steps from the established efficacy of a disease control tool (drug, vaccine with an efficacy of $70 \%$ in Figure 1a) to the communities concerned. It leads from $70 \%$ coverage via the proportion of appropriately diagnosed (those to be treated or vaccinated, $80 \%$ ) and the compliance rate of users and providers $(70 \%)$ to community effectiveness. In this example, $\mathbf{2 7 \%}$, or about one-third of the initial efficacy of $70 \%$, is retained as community effectiveness. An effort that is solely directed to improve efficacy from $70 \%$ to $90 \%$ with all the other elements unchanged leads to a community effectiveness of only $35 \%$ which is still only one-third of the initially applied efficacy of $90 \%$ (Figure 1a). On the other hand, Figure $1 \mathrm{~b}$ shows the situation where the efficacy is not improved, but the application of the tool at the community level is emphasized. This results - despite an efficacy of only $70 \%$ - in a community effectiveness of $54 \%$, thus $77 \%$ of the initially introduced efficacy is retained as community effectiveness.

Strengthening the application of tools implies that health managers need timely and accurate information to reach decisions in launching, monitoring and evaluating disease control programmes. Many of the established health information systems are very cumbersome for health personnel, they are therefore poorly utilized, the results are frequently not fed back to the potential users and therefore cannot be applied at the local level (Tanner 1988). With regard to data use in health service management, the traditional methods of obtaining epidemiological, sociological and anthropological information are often beyond the limits of available resources in terms of time, costs and staff needs. This clearly highlights the potential role of rapid assessment methods in improving the community effectiveness and sustainability of health interventions, and of many other interventions that have community development as their aim.

\section{The nature, development and validation} of rapid assessment

Despite the fact that the term rapid assessment denotes a rather broad collection of techniques in 

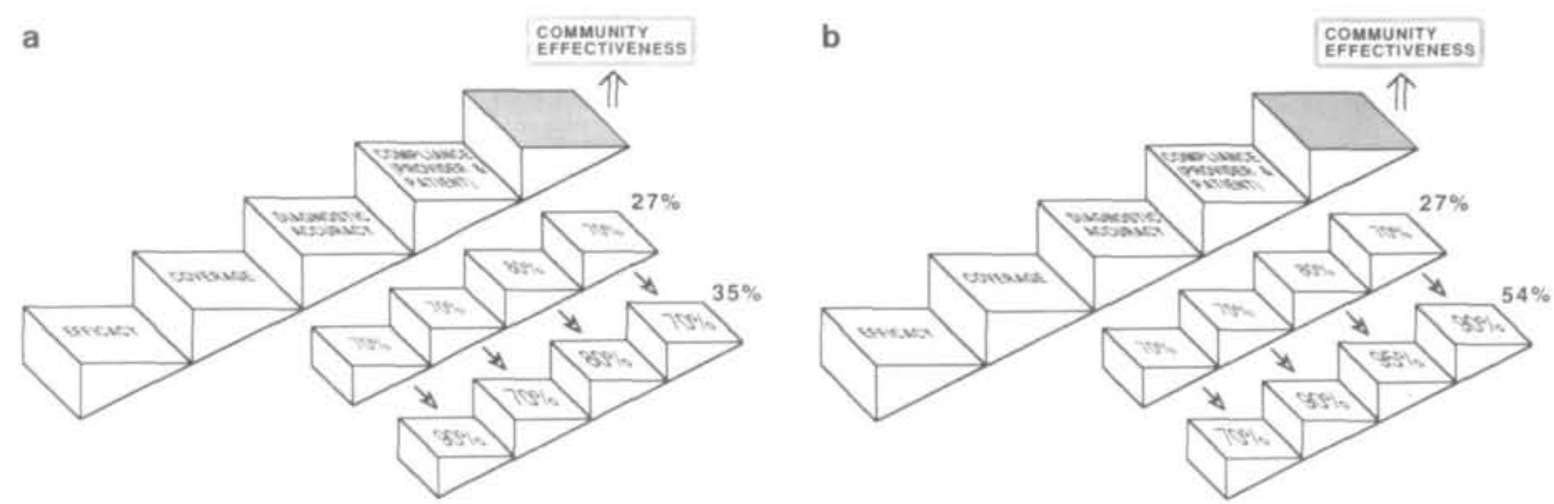

Figure 1. The path from the efficacy of a disease control tool to its community effectiveness is dependent on coverage, the diagnostic accuracy and the compliance of users and providers (based on Tanner 1990; Tugwell et al. 1985). 1a. Situation in which efficacy of the disease control tool is improved from $70 \%$ to $90 \%$. 1b. Situation in which efficacy of disease control tool remains unchanged at $70 \%$, but proportion of accurate diagnosis, compliance and coverage increase through strengthened application of tool at community level.

epidemiology, social science and anthropology, there are a few common features that need to be emphasized. It is particularly important to refute the notion that rapid assessment generates inaccurate data or just summarizes impressions.

Taking into account the need for increasing community effectivenes, it is expected that a rapid assessment procedure will generate qualitative and/or quantitative information. This information can be fed into the health planning cycle, virtually at all levels. It may be used to generate health programme objectives and to assist in planning, implementation and evaluation of the programme. Rapid assessment must provide this information. within a time frame appropriate to the needs and sequences of health care management in a given area.

The users of rapid assessment methods - once developed and validated by biomedical, social science and epidemiological research - are health planners and managers, local level decision makers and community members. Within the given context of application, 'rapid' will be defined by the users. From our perspective, 'rapid' should refer to the period from the use of the method for data collection to the onset of the application of results in the relevant programmes. Thus, 'rapid' should not only refer to the period of the data collection phase. In addition, it needs to be emphasized that the development of rapid assessment may need considerable time; possibly even more than the application of traditional epidemiological, anthropological and social science methods, as careful validation procedures need to be established. Rapid assessment, like other procedures, should aim for the highest possible accuracy.

Validation will always have to focus on this and ensure that a new method or approach in fact measures what it is supposed to measure. On the other hand, precision is always dependent on the objectives of use of the rapid assessment procedure. As indicated below, there will be situations where one does not require high precision. This, in turn, has implications for the sample size needed, and consequently, for the resources required. There are also no all-encompassing definitions for the terms 'simple' and 'cheap'. These dimensions can only be assessed within the context where rapid assessment methods are applied. For example, one might invest substantial resources in rapid assessment methods if they can really improve community effectiveness, and the outcome and impact of health interventions. Therefore, the important criteria will be costeffectiveness and cost-benefit.

The development of rapid assessment methods entails many crucial steps. Besides requiring a careful validation process that ensures the accuracy of the data collected, the operational steps of any rapid assessment procedure must be assessed with respect to the feasibility of its use within existing services. The development of a procedure and its validation is the result of 
in-depth research. The next step is an evaluation of its feasibility within relevant programmes, and its routine use in existing services. For example, verbal autopsies may be extremely useful to assess the prevalence of mortality from a given disease (Snow and Marsh, this issue), but may not be practical for routine use in health services. On the other hand, the questionnaire approach to establish disease control priorities for communities, based on demand patterns (Lengeler et al. this issue), can be applied within an existing administrative structure at district level. To summarize, the development of rapid assessment methods requires not only validation against traditional techniques in epidemiology and the social sciences, but a critical evaluation of the scope and limitations of their application within a given setting.

\section{The application of rapid assessment to tropical diseases}

\section{Potential uses of rapid assessment}

In tropical disease research, rapid assessment methods could be of considerable use in at least five important areas:

(1) First, better estimates of the prevalence of infection and particularly, disease, of virtually all tropical diseases are needed. These are diseases of poverty prevailing among rural populations who have poor access to health services, and hence these are frequently underreported or misreported. Malaria is perhaps the most glaring example of this situation. Not only do these problems prevail, but malaria is often selftreated, even in areas where health facilities exist. Also, malaria is easily confused with fever and other diseases, and in some areas it may even be overreported. In addition, malaria is sometimes associated with activities such as smuggling and illegal lumbering, so that many malaria patients are highly mobile, buying drugs where they can get them, and completely bypassing health systems.

Similarly, but for different reasons, diseases such as leprosy or filariasis are often underreported because of the fear and stigma associated with these afflictions. Better methods of estimating and monitoring disease prevalence are therefore required, and large-scale epidemiological surveys are expensive and time-consuming. Rapid assess- ment methods, preferably repeatable ones, could therefore fill an important role in providing information quickly, and for larger populations.

(2) A second area for which rapid assessment could be most valuable is in providing better estimates of causes of death. In many developing countries, vital registration data on deaths are incomplete, especially in poor rural areas where the TDR target diseases prevail. While morbidity from some of the tropical diseases (filariasis, leprosy, schistosomiasis, leishmaniasis) is a more serious problem than mortality, malaria is among the principal causes of death in many endemic areas, particularly among infants and children. Discussions at a workshop on rapid assessment, held in Baroda in early 1991 (see below), revealed that information about mortality due to Chagas' disease is also deficient, as deaths are often recorded as 'sudden cardiac arrest'. Even in hospitals and clinics, diagnosis of deaths from Chagas' disease may be inaccurate, because medical practitioners generally attribute sudden death in an endemic area to Chagas' disease, sometimes without corroborating biomedical evidence.

Having better estimates of mortality by cause is important not only because current knowledge is poor, but also because we would be able to assess the impact of interventions such as bednets on mortality from malaria, or in the case of Chagas' disease, of housing improvements or the use of insecticides in rural areas. Moreover, we would have a more rational basis for evaluating the importance of given diseases within the overall health problems of communities, and the appropriateness of the attention currently being paid to them. If rapid methods were available to obtain better estimates of causes of death, they could provide the basis for monitoring, assessing and reassessing health needs and priorities on a more regular basis.

(3) Another purpose for which rapid assessment methods are urgently needed is the assessment of high risk situations, or the identification of particular groups at risk of contracting tropical diseases. Most attention has perhaps been given to preventing and evaluating needs in the face of epidemics, political strife and natural disasters, but techniques are also needed to provide more accurate and timely assessments of 
changing morbidity profiles and disease control strategies.

All too often, experts are called upon to provide advice in epidemic situations or in emergencies (for example, where drug resistance has developed, and therapeutic alternatives are limited) and, having little experience or knowledge of the geographical, ecological, sociopolitical and other characteristics of the areas to which they are called, arrive at inaccurate or incomplete assessments of the problem. This implies the need for development of assessment tools which can be applied with more precision and more comprehensively by disease control programmes themselves, both in emergencies and in an ongoing routine within existing services.

(4) Rapid assessment would also be extremely useful in situations where interventions are about to be introduced. It is now well recognized that without an understanding of local conditions, people's beliefs, practices and needs, modern technologies may not be used, and the chances of their ever being used may be further jeopardized by their inappropriate promulgation. One example was the introduction of the intrauterine contraceptive device (IUD) in India in the 1960s, which failed to take account of local needs and concerns (Vlassoff 1979). The tremendous groundswell of negative opinion which followed as a result of people's poor understanding about the method and its side-effects, crippled IUD use to such an extent that it has never been widely accepted in India. Similarly, rapid assessment could provide a basis for the selection of 'experimental' and 'control' communities for intervention studies.

(5) Rapid assessment could also be applied to monitoring and surveillance of ongoing disease control programme activities, community participation or other interventions. Incorporating such methods into the community health care delivery system could result in the maintenance of up-to-date records of the health status of populations, the effectiveness of disease control activities, particular problems experienced with respect to these activities, and other relevant matters.

The use of rapid assessment in monitoring and evaluation also implies the development of approaches to speed up data analysis. The process of data entry and analysis in the field not only makes the research process more reliable, efficient and timely, but also ensures its closer link with the users and thus, with the translation of results into application.

Many questions remain, however, about social and economic aspects of the transmission and control of communicable diseases which do not seem easily amenable to rapid assessment methods. While a number of methods have been, or are being, developed to measure health status and health impact (Table 1), rapid assessment methods in the fields of health service and health behaviour information are lacking, and still require careful consideration. For example, the discussion of rapid anthropological procedures (Manderson and Aaby, this issue) shows that where an in-depth understanding of cultural factors or sensitive topics (including personal fears and stigma associated with diseases such as leprosy) is advisable, rapid assessment would seem much less appropriate than more traditional, longer-term studies.

\section{The Baroda workshop}

In January 1991, a five-day workshop on rapid assessment methods was organized by the Centre for Operations Research and Training and TDR/SER in Baroda, India. As TDR is a research programme, its role in the field of rapid assessment procedure is primarily in the validation of these methods. The objectives of the workshop were therefore to review existing rapid assessment methods in health and related fields; to assess their strengths and weaknesses and; to consider their application to TDR's target diseases. A further objective was the development of research protocols to expand the validation of selected rapid assessment methods already applied to some diseases, to other tropical diseases.

It is essential that SER-funded research be relevant to disease control; hence the workshop included participants from many disciplines (social sciences, epidemiology, entomology, public health and biostatistics) and from academic and disease control programmes. Teams involved in the preparation of research protocols consisted of a social scientist and medical scientist, both closely connected with national disease control 
Table 1. Rapid assessment methods in health by status of TDR applications

\begin{tabular}{|c|c|c|c|c|}
\hline \multirow[t]{2}{*}{ Method } & \multicolumn{4}{|c|}{ Information/purpose to which method addressed } \\
\hline & Health status & Health impact & Health services & Health behaviour \\
\hline Community questionnaires & O & O & $\mathbf{P}$ & \\
\hline Verbal autopsies & o & 0 & & \\
\hline $\begin{array}{l}\text { Rapid assessment } \\
\text { procedures (RAP) }\end{array}$ & F & $\mathbf{F}$ & $\mathbf{F}$ & $\mathbf{F}$ \\
\hline $\begin{array}{l}\text { Rapid data analysis } \\
\text { in field }\end{array}$ & $\mathrm{F}$ & $\mathbf{F}$ & $\mathrm{F}$ & $\mathbf{F}$ \\
\hline $\begin{array}{l}\text { Malaria paradigms } \\
\text { Other rapid epidemiological } \\
\text { assessment methods }\end{array}$ & $\mathbf{F}$ & $\mathbf{P}$ & $\mathbf{F}$ & $\mathbf{P}$ \\
\hline
\end{tabular}

$O=$ Ongoing research $F=$ Future exploration planned $P=$ Possible exploration

activities. Participation of control programmes is key to the ultimate success of rapid assessment methods because it helps to ensure that the research questions being formulated are relevant to local health concerns, and that their results are useful.

The rapid assessment methods investigated at the workshop included:

- rapid community assessment using simple questionnaires and existing administrative systems

- verbal autopsies

- structured morbidity interviews

- rapid assessment procedures ('RAP')

- rapid data collection using hand-held computers

- malaria paradigms.

In addition to an examination of the strengths and weaknesses of these methods and their potential application to tropical diseases, plans for the further validation of these methods were also discussed, and research protocols developed for this purpose. The remaining sections of this paper introduce these methods and outline future plans for their development within the SER programme. The subsequent papers in this issue are revised versions of papers presented at the workshop, with an additional paper by Singer and Sawyer.

\section{Applications of rapid assessment described in this issue}

Table 1 provides a typology of the kinds of research to which rapid assessment has been applied. This is intended as a working tool, and although it includes all the types of rapid assessment of which we are aware, it does not pretend to be complete. The methods described in this issue include only a few of the methods listed in Table 1; that is, those already being validated by TDR (category ' $O$ ' for 'ongoing') and those which were discussed at the workshop and are likely to be investigated by TDR in the near future (category ' $F$ ' for 'future exploration'). Other methods, classified under 'other rapid epidemiological assessment methods', include a range of techniques consisting of sampling, surveillance methods, screening and individual risk assessment, community indicators of risk or health status, and case-control methods for evaluation.

The first rapid assessment method to be described in this issue is the use of 'mailed' community questionnaires, or questionnaires sent through an existing administrative system other than the health system. The method was developed by researchers at the Swiss Tropical Institute to investigate community perceptions of the prevalence and public health importance of urinary schistosomiasis. The questionnaires were designed for key community informants (leaders, teachers, school children) and were not administered personally by the research team. This approach proved highly cost-effective at district level in rural Tanzania, for targeting disease control activities on high risk areas at only a fraction of the cost of laboratory examinations. For example, the cost of the school questionnaires alone in Kilosa District was US\$0.07 per child 
as compared to US $\$ 2.00$ using urine filtration screening (Lengeler et al. 1991). The extension of this approach to test its applicability to different endemic and sociocultural settings in several African countries is described in this issue (details in Lengeler et al). The Baroda workshop explored the applicability of the method to onchocerciasis in Nigeria where the Nigerian Onchocerciasis Control Programme (NOCP) is embarking on a major distribution campaign for ivermectin, a drug which has excellent prospects for the control of river blindness. If the approach proves viable, it could be an extremely costeffective way for the NOCP to determine high risk areas on which to target disease control activities.

Another example of rapid assessment methods which SER proposes to adapt to selected tropical diseases is the verbal autopsy. This method, described in Snow and Marsh (this issue), uses what people say about a disease to develop simple algorithms for attributing probable causes of death. The method appears to have considerable potential for improving estimates of mortality from tropical diseases, in areas where medical records are incomplete or non-existent (Gray et al. 1990). Its use for determining deaths due to malaria is currently being investigated by Snow and his colleagues in Kilifi, Kenya. As a result of the workshop, a study is being designed to test the applicability of this approach to Chagas' disease in selected areas of Latin America.

A similar approach to the verbal autopsy is the structured morbidity interview which, while widely used in research on developing country health problems, has rarely been validated. Kalter (this issue) discusses approaches to validation, factors affecting validation and problems of developing appropriate instruments for use in the field.

Singer and Sawyer (this issue) describe the application of the structured morbidity interview to a single disease - malaria - using reports by the affected population either of their personal experiences or of those of a member of their household. While 'self-reporting' of malaria episodes is a frequently used approach to rapidly assess malaria prevalence and incidence, its per- formance is not often validated by comparisons with epidemiological measures. The results of the Singer and Sawyer paper indicate that this approach has considerable merit, and deserves further attention in future research.

The papers by Manderson and Aaby and Khan and Manderson describe anthropological methods of rapid assessment which have been widely applied elsewhere (nutrition, diarrhoeal diseases, acute respiratory infections) and which, with modifications, could be applied to tropical diseases. These two papers are analytical, taking a critical look at rapid anthropological procedures (RAP) (sometimes referred to as rapid ethnographic assessment), and focus groups, within the context of traditional anthropology.

The final method described in this issue is the use of hand-held computers for rapid data analysis in the field. It demonstrates the costeffectiveness of direct data entry into hand-held computers during the data collection process. This method, developed for one author's doctoral research, is new and has not been applied to any of the TDR diseases. However, we are actively exploring ways of incorporating this approach into different field research situations.

\section{Next steps in the development of rapid assessment methods}

As noted above, several of the methods discussed in this issue are currently being investigated through field studies funded by TDR. Others have not yet been explored in any detail with respect to their application to TDR's target diseases. SER will continue to explore the new methods of rapid assessment which currently exist and, in cases where straightforward application is problematic, their possible adaptation. The latter seems to be the case with the rapid anthropological procedures (RAP) method. A working group of the participants from the Baroda meeting is currently devoting considerable effort to the adaptation of RAP for use on tropical diseases, and at the same time exploring methods to assure increased validity and applicability. We therefore anticipate that an important part of our future work on rapid assessment in tropical diseases will be the expansion of activities in this area. 


\section{References}

Gray RH, Smith G and Barass P. 1990. The use of verbal autopsies to determine selected causes of death in children. Johns Hopkins University School of Hygiene and Public Health. Occasional Paper Number 10.

Lengeler $C$, Kilima $P$, Mshinda $\mathbf{H}$, Morona $D$, Hatz $C$ and Tanner M. 1991. Rapid, low-cost two-step method to screen for urinary schistosomiasis at the district level: the Kilosa experience. Bulletin of the World Health Organization 69: 176-89.

Tanner M. 1988. District level data collection and use. Commissioned paper for International Commission on Health Research for Development. Harvard School of Public Health, Cambridge, Mass.

Vlassoff C. 1979. Fertility control without modernization: evidence from a rural Indian community. Journal of Biosocial Science 11(3): 325-39.

World Health Organization. 1988. From Alma-Ata to the year 2000: a midpoint perspective. Riga, WHO documents (n/d).

\section{Biographies}

Carol Vlassoff, PhD, is Secretary of Social and Economic Research, Special Programme for Research and Training in
Tropical Diseases (TDR), World Health Organization (WHO). She is trained in demography, sociology and philosophy. For the past 12 years she has worked in the field of international development assistance, first with the International Development Research Centre as Associate Director, Population and Development, and now with TDR in the health social science field. Her main task in TDR is the promotion and strengthening of social and economic research for the control of tropical diseases.

Marcel Tanner, BSc, MSc, MSc, PhD, is head of the Department of Public Health and Epidemiology at the Swiss Tropical Institute, Basel, Switzerland, and Associate Professor at the University of Basel. After laboratory-based research on the immunology of filariasis and the in vitro cultivation of trypanosomes he has conducted epidemiological, operational and health systems research in various African countries during the last 10 years. Besides research and teaching in the field of epidemiology and public health, he directs major health service development co-operation programmes in Chad and Tanzania.

Correspondence: Dr Carol Vlassoff, Social and Economic Research, Special Programme for Research and Training in Tropical Diseases, World Health Organization, 1211 Geneva 27, Switzerland. 